\title{
Endovascular treatment for pseudoaneurysm after carotid blowout syndrome
}

Chong Hyuk Chung, MD'; Young-Nam Roh, MD, PhD²; Seo Hyeon Lee, $\mathrm{MD}^{3}$; Yeong Seok Jeong, $\mathrm{MD}^{3}$; Jeong-Ho Hong, $\mathrm{MD}, \mathrm{PhD}^{3}$; Sung-Il Sohn, $\mathrm{MD}, \mathrm{PhD}^{3}$; Hyungjong Park, $\mathrm{MD}^{3}$

'Division of Rheumatology, Department of Internal Medicine, Wonkwang University College of Medicine, Iksan, Republic of Korea

${ }^{2}$ Department of Surgery, Keimyung University School of Medicine, Daegu, Republic of Korea ${ }^{3}$ Department of Neurology, Keimyung University School of Medicine, Daegu, Republic of Korea

\section{Journal of Neurocritical \\ Care}

CASE REPORT

Received: September 24, 2020

Revised: November 9, 2020

Accepted: November 26, 2020

Corresponding Author:

Hyungjong Park, MD

Department of Neurology, Keimyung

University School of Medicine, 1035

Dalgubeol-daero, Dalseo-gu, Daegu

42601, Korea

Tel: +82-53-258-7747

Fax: +82-53-258-4380

E-mail: hjpark209042@gmail.com

Background: Pseudoaneurysms of the carotid artery are rare and can be both a cause and consequence of carotid blowout syndrome (CBS) in patients with head and neck cancer. Surgical or endovascular treatment approaches may be useful for managing this condition.

Case Report: A 55-year-old man presented with a pulsatile mass surrounding the carotid bifurcation in the neck. He reported a history of CBS that occurred as a surgical complication while removing a benign neck cyst. Additionally, he reported a history of laryngeal cancer that underwent complete remission after surgery and radiotherapy. The mass was diagnosed as a pseudoaneurysm. Surgical treatment proved challenging owing to the patient's history of neck surgery and radiotherapy; therefore, stent-graft deployment was performed and the absence of pulsations was checked through the neck mass.

Conclusion: Endovascular treatment may be a useful therapeutic strategy in cases of post-CBS pseudoaneurysm in patients undergoing surgery and/or radiotherapy for head and neck cancer.

Keywords: Aneurysm, False; Carotid artery injuries; Stents

\section{INTRODUCTION}

Carotid artery (CA) aneurysms are rare and account for approximately $0.4 \%-4 \%$ of all peripheral artery aneurysms [1]. Pseudoaneurysms are the most common type of CA aneurysms [2]. Trauma is the most common cause of CA pseudoaneurysm, followed by surgery and/or radiotherapy for head and neck cancer [3]. Pseudoaneurysms are not covered by healthy, well-vascularized tissue and can therefore rupture easily [4]. Carotid blowout syndrome (CBS) is the rupture of the CA and its branches, which can cause life-threatening complications due to massive blood loss, as well as neurological complications such as cerebral infarction [5]. A pseudoaneurysm of the CA, while being one of the main known causes of CBS, can also occur as a surgical complication for CBS $[1,6]$.

Pseudoaneurysms are treated using open surgery or with endovascular treatments, such as parent vessel sacrifice, using detachable balloons or coil embolization and stent-graft placement $[7,8]$.

(C) 2020 The Korean Neurocritical Care Society

This is an Open Access article distributed under the terms of the Creative Commons Attribution Non-Commercial License (http://creativecommons.org/licenses/by-nc/4.0/) which permits unrestricted noncommercial use, distribution, and reproduction in any medium, provided the original work is properly cited. 
Currently, endovascular treatment is regarded as a better alternative to open surgery in selected patients [7]. We report a case of post-CBS pseudoaneurysm that was treated successfully using endovascular therapy in a patient with a history of surgery and radiotherapy for laryngeal cancer.

\section{CASE REPORT}

A 55-year-old man was diagnosed with laryngeal cancer 23 years prior to presentation; complete tumor resolution was observed after partial laryngectomy and radiotherapy. Approximately 1 year before his visit, a palpable mass surrounding the left carotid bifurcation was observed. Ultrasonography revealed a subcutaneous, solid, and well-demarcated ovoid mass that distorted the left internal jugular vein thus necessitating excision. The left internal CA (ICA) was injured intraoperatively during neck dissection which resulted in active bleeding that was managed by an emergency primary suture at the site of injury. The following day, the patient was observed to be drowsy, with an altered mental status, and with severe dysarthria. Diffusion-weighted magnetic resonance imaging revealed border-zone and cortical infarctions in the left cerebral hemisphere (Fig. 1A and B). Computed tomography angiography (CTA) showed multifocal long-segment stenosis of the left common CA (CCA) (Fig. 1C). Volume replacement was performed in addition to blood pressure control to maintain cerebral perfusion pressure. The patient showed complete recovery a month later and was discharged with aspirin $(100 \mathrm{mg})$, clopidogrel (75 $\mathrm{mg})$, and atorvastatin $(80 \mathrm{mg}$ ) for multifocal stenosis extending along the CCA to the ICA. Histopathologic findings of the neck mass revealed benign soft tissue without any evidence of inflammation or tumor cells. No bleeding or abnormal vessel formation was observed at the site of CBS; the patient was therefore instructed to follow up with the outpatient department.

During follow-up, a pulsating mass was detected in the vicinity of the previous site of injury (Fig. 1D). This mass gradually increased in size and was clearly identified a year after the development of the CBS. CTA revealed a new pseudoaneurysm immediately beneath the skin in addition to multifocal long-segment stenosis of the left CCA (Fig. 1E). Because of the patient's history of partial laryngectomy and radiotherapy, we expected that performing surgical ligation would be technically challenging; therefore, a self-expandable stent graft was used to treat the post-CBS pseudoaneurysm. We inserted a 10-F sheath through the right femoral artery and an 8-F guiding catheter (Shuttle; Cook, Bloomington, IN, USA) was into the proximal portion of the left CCA. Left CCA angiography revealed a pseudoaneurysm at the carotid bulb with multifocal long-segment stenosis of the left CCA (Fig. 1F).
A microcatheter (Headway 17; Microvention, Tokyo, Japan) was placed at the orifice of the external CA (ECA) and coil embolization (Tornado, Cook) of the left ECA was performed to prevent a type II endoleak after stent placement. A 0.035-inch guidewire was placed at the petrous portion of the left ICA after confirming that coil embolization had completely blocked blood flow through the ECA. A $9 \times 60-\mathrm{mm}$ self-expandable stent graft (Covera Plus; Bard Medical, Covington, GA, USA) was selected based on the dimensions of the affected CA (the graft size was at least $1 \mathrm{~mm}$ greater than the diameter of the CCA). The self-expandable stent graft was then advanced over the 0.035-inch guidewire and deployed to extend between the distal segment of the left CCA and the proximal ICA, ensuring that the pseudoaneurysm was completely covered. Left CCA angiography was performed after stent graft deployment and showed the absence of a pseudoaneurysm. We subsequently placed a carotid stent into the stenotic segment of the left CCA, which, if left untreated, could have served as a potential source of emboli thereby predisposing the patient to ischemic stroke. A $9 \times 50 \mathrm{~mm}$ carotid stent (Carotid Wallstent; Boston Scientific, Santa Clara, CA, USA) was advanced over a 0.014inch microwire (Transend; Stryker, Kalamazoo, MI, USA) and deployed from immediately beneath the orifice of the left proximal ICA, partially overlapping the previously deployed self-expandable stent graft. Left CCA angiography revealed no pseudoaneurysms, in-stent stenosis, or in-stent thrombosis (Fig. 1G). Carotid duplex ultrasonography was performed the following day and revealed no stenotic flow, in-stent stenosis, or thrombosis. Moreover, the patient did not show any postoperative neurological symptoms. A CTA was performed 7 days postoperatively which confirmed the disappearance of the pseudoaneurysm with good stent patency (Fig. 1H). After 3 months, the pulsatile mass disappeared completely (Fig. 1I).

\section{DISCUSSION}

A pseudoaneurysm is defined as a loss of integrity of the three layers of the arterial wall that can result in a contained rupture of blood vessels; this differs from a true aneurysm in which the three vascular walls are intact but dilated [9]. Pseudoaneurysms of the ICA are known to present as a growing pulsatile mass in the lateral aspects of the neck [10].

Radiotherapy following head and neck cancer surgery is known as an important cause of CA pseudoaneurysm that can lead to CBS [11]. The CA receives $80 \%$ of its blood supply from the vasa vasorum in its adventitia (the outermost layer of the CA) [4]; radiotherapy results in injury to the adventitia of the $\mathrm{CA}$ and/or its major branches, resulting in the weakening of the arterial wall 

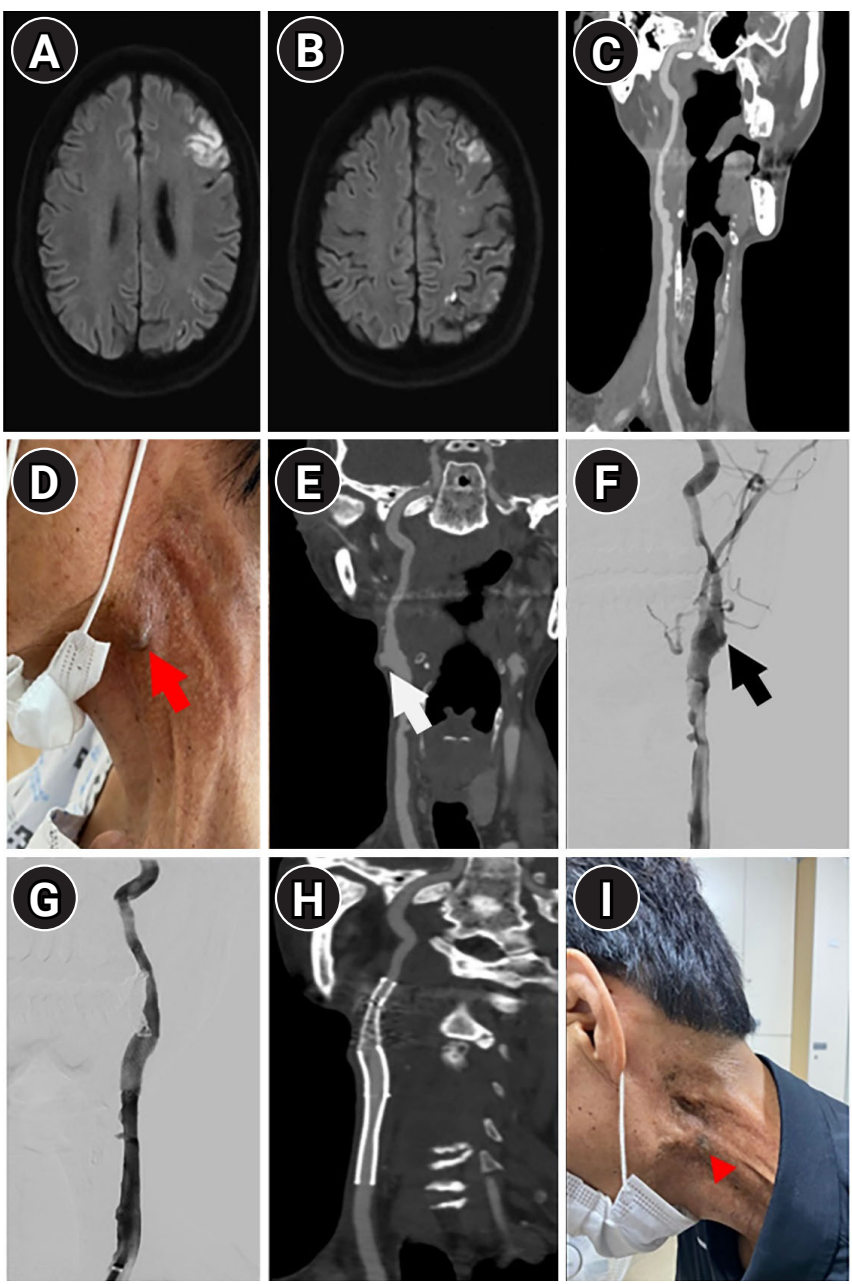

Fig. 1. (A, B) Cortical and border-zone infarctions in the left cerebral hemisphere. (C) Multifocal long-segment stenosis of the left common carotid artery extending to the proximal internal carotid artery on computed tomography angiography. (D) A pulsating mass in the vicinity of the previous injury site of the carotid artery (red arrow). (E) Pseudoaneurysm immediately beneath the skin (white arrow). (F) Pseudoaneurysm (black arrow) with long-segment stenosis of the left common carotid artery on digital subtraction angiogram. (G) Non-visualization of pseudoaneurysm after deployment of a self-expandable stent graft. $(\mathrm{H})$ The disappearance of the pseudoaneurysm with good stent patency on computed tomography angiogram 7 days after endovascular treatment. (I) The disappearance of the pulsatile mass in the vicinity of the previous injury site of the carotid artery (red arrowhead) after 3 months.

$[5,12]$. Thus, radiotherapy itself can be the main cause of pseudoaneurysm formation in CA. In addition, weakening of the arterial wall due to radiotherapy after head and neck cancer surgery can increase the risk of pseudoaneurysm formation after surgical repair for CBS.

Treatment modalities for pseudoaneurysms can be categorized into surgical repair and endovascular treatment $[7,8]$. Surgical re- pair involves surgical ligation of the affected ICA followed by vascular bypass or anastomosis; this procedure sacrifices the ICA. Furthermore, surgical ligation can predispose patients to cerebral ischemia, and bypass or anastomosis cannot be performed when the aneurysms are located near the skull base. Additionally, the open access approach is challenging in patients who previously underwent surgery or radiotherapy for head and neck cancer [8].

Endovascular treatment involves deconstructive methods, such as occlusion of the affected ICA using a balloon or coil, and reconstructive methods, such as stent-graft placement $[5,7]$. Because stent-graft placement is less invasive and has lower complication rates [13], it may be a useful strategy for managing post-CBS aneurysms especially in patients with a history of surgery and/or radiotherapy for head and neck cancer. Nevertheless, the following factors should be considered prior to stent-graft placement for CBS: (1) it is necessary to assess the patient's anatomical suitability for the procedure, as this method is only feasible in patients without excessive vascular tortuosity, to ensure easy access for the stent delivery system, and in those with patent femoral or iliac arteries to ensure placement of a large-caliber $(10-11 \mathrm{~F})$ vascular sheath [14]; (2) stent-grafts are more thrombogenic than bare stents and warrant long-term aggressive antiplatelet therapy which involves a high risk for bleeding complications [15]; and (3) endoleak is a common complication of stent-graft placement. Specifically, reconstitution of the proximal branches of the ECA for lesions in the vicinity of the carotid bifurcation is associated with a risk for developing a type II endoleak from collateral vessels [16]; however, embolization of the main trunk of the ipsilateral ECA can reduce this risk [14].

In our case, the patient was relatively young and had a favorable vascular anatomy and was therefore considered to be a suitable candidate for endovascular treatment. In addition, severe adhesions were detected intraoperatively between the CA and the surrounding connective tissue, which occurred at the time of CBS development. Based on these findings, we performed endovascular treatment for a post-CBS pseudoaneurysm. Furthermore, we performed ECA embolization to prevent a type II endoleak.

In conclusion, the anatomical constraints associated with surgical treatment of post-CBS pseudoaneurysm make endovascular treatment an appealing alternative. This case illustrates the usefulness of stent-graft placement in treating post-CBS pseudoaneurysms.

\section{ARTICLE INFORMATION}

\section{Ethics statement}

This study was reviewed and approved by the Institutional Re- 
view Board of Keimyung University Dongsan Hospital (IRB No. 2020-09-068). The requirement to obtain informed consent was waived by the Board.

\section{Conflict of interest}

Dr. JH Hong is an editorial board member of the journal but was not involved in the peer reviewer selection, evaluation, or decision process of this article. There are no other potential conflicts of interest relevant to this article to declare.

\section{Funding}

This work was supported by a National Research Foundation of Korea (NRF) grant funded by the Korean government (MSIT) (No. 2020R1G1A007100).

\section{ORCID}

Chong Hyuk Chung https://orcid.org/0000-0001-5806-0733 Young-Nam Roh https://orcid.org/0000-0002-0799-1578

Seo Hyeon Lee https://orcid.org/0000-0001-8357-8956

Yeong Seok Jeong https://orcid.org/0000-0003-4778-7413

Jeong-Ho Hong https://orcid.org/0000-0002-8235-9855

Sung-Il Sohn https://orcid.org/0000-0002-6900-1242

Hyungjong Park

\section{Author contributions}

Conceptualization: HP, Data curation: CHC, YNR, SHL, YSJ, JHH, SIS, Writing-original draft: CHC, HP, Writing-review \& editing: HP.

\section{REFERENCES}

1. Faggioli GL, Freyrie A, Stella A, Pedrini L, Gargiulo M, Tarantini S, et al. Extracranial internal carotid artery aneurysms: results of a surgical series with long-term follow-up. J Vasc Surg 1996;23:587-94.

2. Bellosta R, Sesana M, Baglini R, Luzzani L, Talarico M, Sarcina A. Endovascular treatment of a symptomatic carotid artery aneurysm with a stent graft. Vasc Endovascular Surg 2008;42:2768.

3. Li Z, Chang G, Yao C, Guo L, Liu Y, Wang M, et al. Endovascular stenting of extracranial carotid artery aneurysm: a systematic review. Eur J Vasc Endovasc Surg 2011;42:419-26.

4. Citardi MJ, Chaloupka JC, Son YH, Ariyan S, Sasaki CT. Management of carotid artery rupture by monitored endovascular therapeutic occlusion (1988-1994). Laryngoscope 1995;105:

1086-92.

5. Chaloupka JC, Putman CM, Citardi MJ, Ross DA, Sasaki CT. Endovascular therapy for the carotid blowout syndrome in head and neck surgical patients: diagnostic and managerial considerations. AJNR Am J Neuroradiol 1996;17:843-52.

6. Lee CW, Yang CY, Chen YF, Huang A, Wang YH, Liu HM. CT angiography findings in carotid blowout syndrome and its role as a predictor of 1-year survival. AJNR Am J Neuroradiol 2014; 35:562-7.

7. Troutman DA, Mohan CR, Samhouri FA, Sohn RL. Endovascular repair of carotid artery pseudoaneurysm after carotid endarterectomy with self-expanding covered stents-a long-term follow-up. Ann Vasc Surg 2010;24:954.e13-6.

8. Attigah N, Külkens S, Zausig N, Hansmann J, Ringleb P, Hakimi $\mathrm{M}$, et al. Surgical therapy of extracranial carotid artery aneurysms: long-term results over a 24-year period. Eur J Vasc Endovasc Surg 2009;37:127-33.

9. Pearson SE, Choi SS. Pseudoaneurysm of the internal carotid artery: a case report and review of the literature. Arch Otolaryngol Head Neck Surg 2005;131:454-6.

10. Grossi RJ, Onofrey D, Tvetenstrand C, Blumenthal J. Mycotic carotid aneurysm. J Vasc Surg 1987;6:81-3.

11. Chaloupka JC, Roth TC, Putman CM, Mitra S, Ross DA, Lowlicht RA, et al. Recurrent carotid blowout syndrome: diagnostic and therapeutic challenges in a newly recognized subgroup of patients. AJNR Am J Neuroradiol 1999;20:1069-77.

12. Lesley WS, Chaloupka JC, Weigele JB, Mangla S, Dogar MA. Preliminary experience with endovascular reconstruction for the management of carotid blowout syndrome. AJNR Am J Neuroradiol 2003;24:975-81.

13. Bond KM, Brinjikji W, Murad MH, Cloft HJ, Lanzino G. Endovascular treatment of carotid blowout syndrome. J Vasc Surg 2017;65:883-8.

14. Chang FC, Lirng JF, Luo CB, Guo WY, Teng MM, Tai SK, et al. Carotid blowout syndrome in patients with head-and-neck cancers: reconstructive management by self-expandable stentgrafts. AJNR Am J Neuroradiol 2007;28:181-8.

15. Tielliu IF, Verhoeven EL, Zeebregts CJ, Prins TR, Span MM, van den Dungen JJ. Endovascular treatment of popliteal artery aneurysms: results of a prospective cohort study. J Vasc Surg 2005;41:561-7.

16. Chang FC, Lirng JF, Luo CB, Wu HM, Guo WY, Teng MM, et al. A self-expandable stent overlapped with a stent-graft as a cause of endoleak in a patient with carotid blowout syndrome. Clin Radiol 2008;63:948-51. 